[1] Young, N.M., et al., Structure of the N-linked glycan present on multiple glycoproteins in the gram-negative bacterium, Campylobacter jejuni. Journal of Biological Chemistry, 2002. 277(45): p. 42530-42539.

[2] Olivier, N.B., et al., In vitro biosynthesis of $U D P-N, N$ 'diacetylbacillosamine by enzymes of the Campylobacter jejuni general protein glycosylation system. Biochemistry, 2006. 45(45): p. 13659-13669.

\section{MS03 P10}

Hyperthermophilic phosphotriesterase: insights into stability and catalytic mechanism Mikael Elias ${ }^{\mathrm{a}}$, Jérôme Dupuy $^{\mathrm{b}}$, Luigia Merone ${ }^{\mathrm{c}}$, Sébastien Moniot ${ }^{\mathrm{a}}$, Claude Lecomte $^{\mathrm{a}}$, Mosè Rossi ${ }^{\mathrm{c}}$, Patrick Masson ${ }^{\mathrm{d}}$, Guiseppe Manco $^{\mathrm{c}}$ and Eric Chabriere ${ }^{\mathrm{a}},{ }^{\mathrm{a}} L C M 3 B, C N R S$-Universite Henri Poincaré, Nancy, France. ${ }^{\mathrm{b}}$ LCCP, Institut de Biologie Structurale, Grenoble, France, ${ }^{\mathrm{c}} I B P$, Consiglio Nazionale delle Ricerche, Napoli, Italy, ${ }^{\mathrm{d}}$ CRSSA, Grenoble, France

E-mail: mikael.elias@1cm3b.uhp-nancy.fr

Keywords: hyperthermophile, catalytic mechanism, anomalous scattering methods.

Organophosphates (OPs) constitute the largest class of insecticides used worldwide and certain of them are potent nerve agents. Consequently, organophosphates-degrading enzymes are of paramount interest, as they could be used as bioscavengers and biodecontaminants [1]. The most efficient OP-degrading enzymes are phosphotriesterases (PTEs), whose natural substrate(s) still remains unknown. Here we report the first three-dimensional structure at 2.6 $\AA$ resolution of a hyperthermophilic PTE (SsoPox), isolated from the archeon Sulfolobus solfataricus. This enzyme is of exceptional thermostability, as catalytic activity is still increasing over $95^{\circ} \mathrm{C}$ [2]. Structural analysis suggests that the increased stability of this protein is achieved by a number of fine structural differences from the less thermostable homologs. Possible determinants of thermostability in SsoPox PTE are the deletion and the stabilization of flexible regions; the dimer of SsoPox is more compact; an increased number of ion pairs, and their network organization. In addition, the active site region reveals some interesting features. First, the presence of an unexpected hydrophobic channel connected to the active site may represent an important clue in the search of the natural substrate of this enzyme. Second, we investigated the chemical properties of the active site constituted by a bimetallic center. Using crystallography and anomalous scattering properties, we characterized in a non ambiguous way that the active site is constituted of a heterobinuclear cobalt / iron center. Finally, many evidences provided from the structure, mutagenesis experiments, and from previous works on mesophilic PTEs, allowed us to refine the catalytic mechanism of these enzymes.

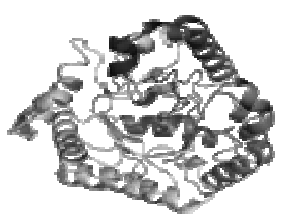

Global structure of SsoPox

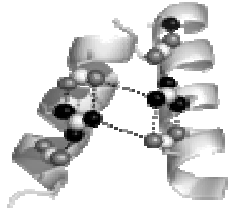

One of the ion pairs networks present in SsoPox structure
[1] LeJeune K.E., Wild J.R., Russell A.J. (1998). Nerve agents degraded by enzymatic foams. Nature 395, 27-8. Merone, L., Mandrich, L., Rossi, M., and Manco, G. (2005). A thermostable phosphotriesterase from the archaeon Sulfolobus solfataricus: cloning, overexpression and properties. Extremophiles 9, 297305.

\section{MS03 P11 \\ Structural Details in the $\beta$-elimination Mechanism of Tyrosine Phenol-lyase Dalibor Milić, ${ }^{a}$ Dubravka Matković-Čalogović, ${ }^{\mathrm{a}}$ Tatyana T. Demidkina, ${ }^{\mathrm{b}}$ Alfred A. Antson, ${ }^{c}$ a Department of Chemistry, Faculty of Science, University of Zagreb, Croatia. ${ }^{\mathrm{b}}$ Engelhardt Institute of Molecular Biology, Russian Academy of Sciences, Moscow, Russia. ' ${ }^{\mathrm{S} S t r u c t u r a l}$ Biology Laboratory, University of York, York, UK}

E-mail: dmilic@chem.pmf.hr

Keywords: tyrosine phenol-lyase, protein-ligand complexes, enzyme mechanisms

Tyrosine phenol-lyase (TPL; EC 4.1.99.2) is a homotetrameric pyridoxal-5'-phosphate (PLP)-dependent enzyme that catalyses the $\beta$-elimination of L-tyrosine (the reversible hydrolytic cleavage of L-tyrosine to phenol and ammonium pyruvate [1]). The $\beta$-elimination proceeds via several intermediate steps, including the cleavage of the $\mathrm{C}_{\beta}-\mathrm{C}_{\gamma}$ bond. In order to reveal details in the enzymatic reaction and understand structural events during the catalysis, we determined the X-ray structures of several different forms of TPL from Citrobacter freundii. All of them are the structures of non-covalent complexes which resemble the quinonoid or the aminoacrylate reaction intermediate. As previously shown [2], the TPL active site can possess two different conformations: open and closed. Our study showed that the proposed closure of the active site during the enzymatic reaction "forces" the quinonoid intermediate into the "strained" conformation, which resembles the transition structure, and thus makes the cleavage of the $\mathrm{C}_{\beta}-\mathrm{C}_{\gamma}$ bond easier. The "strained" conformation of the quinonoid intermediate is stabilised by hydrogen bonding and van der Waals interactions with the active site residues.

[1] Phillips, R.S.; Demidkina, T.V.; Faleev N.G. Biochim. Biophys. Acta, 2003, 1647, 167.

[2] Milić, D.; Matković-Čalogović, D.; Demidkina, T.V.; Kulikova, V.V.; Sinitzina, N.I.; Antson, A.A. Biochemistry, 2006, 45,7544 .

MS03 P12

Crystal structures of conjugates of soman with TcAChE Sanson Benoit ${ }^{\mathrm{a}}$, Nachon Florian ${ }^{\mathrm{b}}$, Colletier Jacques-Philippe $^{\mathrm{c}}$, Silman Israel $^{\mathrm{d}}$, Sussman Joel L. ${ }^{\mathrm{e}}$, Masson Patrick ${ }^{\mathrm{b}}$, Weik Martin, ${ }^{\mathrm{a}}$ Laboratoire de Biophysique Moléculaire, Institut de Biologie Structurale CEA-CNRS-UJF, Grenoble,France, ${ }^{b}$ Unité d'Enzymologie, Département de Toxicologie, Centre de Recherches du Service de Santé des Armées, La Tronche, France, ${ }^{c}$ Molecular Biology Institute, University of California, Los Angeles, USA, Departments of ${ }^{d}$ Neurobiology and ${ }^{e}$ Structural Biology, Weizmann Institute of Science, Rehovot, Israel. E-mail: benoit.sanson@ibs.fr

Keywords: organophosphorus poisons, protein crystallography, acetylcholinesterase

Acetylcholinesterase (AChE) is one of the fastest enzymes in Nature. It hydrolyses the neurotransmitter acetylcholine at $\sim 10000$ times per second, thus achieving its role in terminating neurotransmission at cholinergic synapses. $\mathrm{AChE}$ is the target of organophosphorus (OP) nerve 
agents, anti-Alzheimer drugs, and insecticides. A better understanding of its molecular mode of action thus goes beyond academic interest, and may be beneficial to the design of anti-Alzheimer drugs, antidotes against OP poisoning and novel insecticides. We have solved crystal structures of conjugates with Torpedo californica AChE (TcAChE) of the potent nerve agent, soman. Soman reacts covalently with the active-site serine to form an initial conjugate (the 'non-aged' conjugate), that can be reactivated by suitable oximes. This conjugate subsequently undergoes a dealkylation reaction known as 'aging', that produce a conjugate that can no longer be reactivated (the 'aged conjugate') [1]. X-ray crystallography data collection performed at the ESRF (Grenoble, France), permitted solution of the structure of both conjugates. Since 'aging' reaction is fast at room temperature, the crystal-soaking time prior to cryo-cooling had to be reduced to minutes in order to trap the "nonaged' conjugate. The structures obtained supply a structural basis for rational designing of more oxime reactivators that may serve as antidotes of OP intoxication.

[1] Millard, C.B. et al., "Crystal structures of aged phosphonylated acetylcholinesterase: nerve agent reaction products at the atomic level" Biochemistry 38, $7032-7039$ (1999)

\section{MS03 P13}

Crystallographic study of E. coli WrbA protein in complex with its flavin cofactor Julie Wolfova, ${ }^{\mathrm{a}, \mathrm{b}}$, Jiri Brynda ${ }^{\mathrm{a}, \mathrm{c}}$, Jannette Carey ${ }^{\mathrm{d}}$, Ivana Kuta Smatanova ${ }^{\mathrm{a}, \mathrm{b}}$. Institute of Physical Biology, University of South Bohemia Ceske Budejovice, Nove

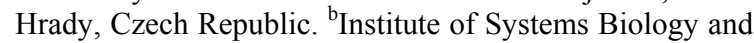
Ecology, Academy of Science of the Czech Republic,

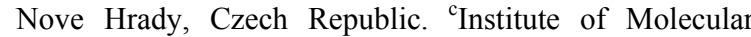
Genetics, Academy of Sciences of the Czech Republic, Prague, Czech Republic. ${ }^{\mathrm{d}}$ Chemistry Department, Princeton University, Princeton, USA.

E-mail: julinka.w@tiscali.cz

\section{Keywords: crystallography of biological macromolecules, flavoproteins, protein-ligand complexes}

The currently studied protein WrbA from Escherichia coli belongs to a new family of multimeric flavoproteins [1,2], that contain flavin mononucleotide (FMN) as a physiological cofactor. The latest observations revealed that E. coli WrbA and its homologues exhibit the $\mathrm{NAD}(\mathrm{P}) \mathrm{H}$ :quinone oxidoreductase activity, which is proposed to be implicated in protection mechanisms against oxidative stress [3]. Crystallographic study of the $E$. coli $\mathrm{WrbA}$ is aimed at proper characterization of the new protein family with respect to its recently revealed oxidoreductase activity. Recombinant WrbA protein was expressed in E. coli CY15071(1DE3) cells and purified as described previously [2]. Pure WrbA protein was obtained without bound cofactor, because of the loss of FMN during purification. The complex of WrbA protein with the FMN cofactor for crystallization was prepared by incubation of pure WrbA with equimolar concentration of FMN. Crystallization was carried out at $285 \mathrm{~K}$ by the sitting-drop vapor-diffusion technique, with droplets containing equal parts of protein and precipitant solution. Yellow, well-formed tetragonal crystals of WrbA protein in complex with its flavin cofactor were obtained from several crystallization conditions. Yielding of diffraction- quality crystals without additional optimzation steps shows the positive influence of FMN cofactor on crystallization of WrbA protein. This effect reflects FMN as a potential stabilizer of the protein structure. Diffraction data for the crystals reported here were collected at $100 \mathrm{~K}$ at synchrotron DESY, beamline X13 (EMBL) with an Xray wavelength of $0.805 \AA$. The different crystallization conditions yielded two forms of primitive tetragonal crystals having different space groups (P43212; P41212) and different unit-cell dimensions $(\mathrm{a}=\mathrm{b}=94.351 \AA$, $\mathrm{c}=$

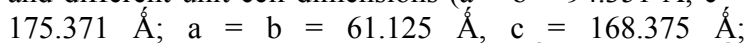
respectively). The resolutions of $2.60 \AA$ and $1.99 \AA$, respectively, were reached. The diffraction limit of the better-diffracting crystal represents the best resolution reported to date for WrbA protein in complex with FMN. Solving of the structures from both data sets is in progress.

This work is supported by the Ministry of Education of the Czech Republic (projects: Kontakt ME640, MSM6007665808, LC06010) and by the Academy of Sciences of the Czech Republic (AV0Z60870520)

[1] Grandori R., Carey J., Protein Sci., 1994, 3, 2185.

[2] Grandori R., Khalifah P., Boice J.A., Fairman R., Giovanelli

K., Carey J., J. Biol. Chem., 1998, 273, 20960.

[3] Patridge E.V., Ferry J.G., J. Bacteriol., 2006, 188, 3498.

\section{MS03 P14}

Carbohydrate-binding of the starch binding domain of Rhizopus oryzae glucoamylase in complex with $\beta$-cyclodextrin and maltoheptaose

Yuj-Ju Sun ${ }^{\mathrm{a}}$, Jung-Yu Tunga ${ }^{\mathrm{a}}$, Yen-Yi Liu ${ }^{\mathrm{a}}$, Wei-I Chou, Margaret D.-T. Chang ${ }^{\mathrm{b}}$, Institute of Bioinformatics and Structural Biology and ${ }^{\mathrm{b}}$ Institute of Molecular and Cellular Biology, National Tsing Hua University, Hsinchu 300, Taiwan, ROC._E-mail: yjsun@life.nthu.edu.tw

Keywords: Rhizopus oryzae glucoamylase, starch binding domain, carbohydrate binding

Glucoamylase hydrolyses starch and polysaccharides to $\beta$ D-glucose. Rhizopus oryzae glucoamylase consists of two functional domains, an N-terminal starch binding domain (SBD) and a C-terminal catalytic domain. The two domains are connected by an O-glycosylated linker. The crystal structures of the SBD in complex with a cyclic carbohydrate, $\beta$-cyclodextrine, and a linear carbohydrate, maltoheptaose, were determined. The overall structures of the SBD complexes belong to a $\beta$-sandwich fold with an immunoglobulin-like structure and two carbohydratebinding sites were observed. Site I is created by several conserved aromatic residues, Trp47, Tyr83, and Tyr94, to form a broad, flat, and firm hydrophobic surface. In addition to the hydrophobic interaction, numerous asparagines residues are involved in the hydrophilic interactions to carbohydrate. Site II is built up by Tyr32 and Phe58 to produce a protruded and narrow binding environment. Site I undergoes a bigger conformational change than site II upon the carbohydrate binding. 